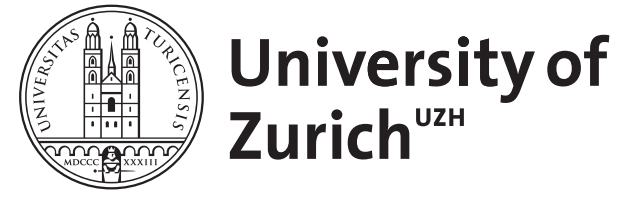

\title{
Some remarks on the distributional Jacobian
}

De Lellis, C

\begin{abstract}
In this paper we prove a strong coarea-type formula and a chain rule for distributional Jacobians of some classes of maps. In particular we give a partial answer to a question arisen by Jerrard and Soner in Jerrard and Soner (Indiana Univ. Math. J. 51 (2002) 645).
\end{abstract}

DOI: https://doi.org/10.1016/S0362-546X(03)00046-4

Posted at the Zurich Open Repository and Archive, University of Zurich ZORA URL: https://doi.org/10.5167/uzh-21889

Journal Article

Originally published at:

De Lellis, C (2003). Some remarks on the distributional Jacobian. Nonlinear Analysis: Theory, Methods Applications, 53(7-8):1101-1114.

DOI: https://doi.org/10.1016/S0362-546X(03)00046-4 


\title{
SOME REMARKS ON THE DISTRIBUTIONAL JACOBIAN
}

\author{
CAMILLO DE LELLIS (SNS ${ }^{1}$ )
}

\begin{abstract}
In this paper we prove a strong coarea-type formula and a chain rule for distributional Jacobians of some classes of maps. In particular we give a partial answer to a question arisen by Jerrard and Soner in [9].
\end{abstract}

Keywords: distributional Jacobians, functions of higher bounded variations, coarea fomula, chain rule

\section{INTRODUCTION}

The starting point of this paper is a "weak" coarea formula involving distributional Jacobians proved by Jerrard and Soner in [9]. The notion of distributional Jacobian is based on the fact that maps $u \in$ $C^{1}\left(\mathbf{R}^{m}, \mathbf{R}^{n}\right)$, with $m \geq n$, satisfy $d\left(u_{1} d u_{2} \wedge \ldots \wedge d u_{n}\right)=d u_{1} \wedge \ldots \wedge d u_{n}$. This identity holds in the sense of distributions for maps $u \in W_{l o c}^{1, p}$ with $p \geq n$ but fails when $p<n$. However if $p \geq n-1$ and $u \in L^{\infty}$ then $\nu=u_{1} d u_{2} \wedge \ldots \wedge d u_{n}$ is an $L^{1}$ function and we can define the distributional Jacobian $[\mathrm{Ju}]$ as the exterior derivative of $\nu$ in the sense of distributions (we refer to [9] for an account of its applications and of the main papers on the argument). We notice that $\nu$ has a natural action (via the classical Hodge operator) as $m-n+1$-dimensional current and we can think of $[J u]$ as the boundary of $\nu$ (see Definitions 2.2, 2.3 and Remark 2.4; for a more general discussion of the properties of $[J u]$ as current we refer to [5]).

If we define $u^{y}=(u-y) /|u-y|$ for every $y \in \mathbf{R}^{n}$ such that $\mathcal{L}^{m}\left(u^{-1}(y)\right)=0$, then we have the following

Theorem 1.1. (See sect. 4 of [9]) If $u \in W^{1, n-1} \cap L^{\infty}$ then $u^{y} \in$ $W^{1, n-1} \cap L^{\infty}$ for a.e. $y \in \mathbf{R}^{n}$ and

$$
\langle[J u], \omega\rangle=\frac{1}{\omega_{n}} \int_{\mathbf{R}^{n}}\left\langle\left[J u^{y}\right], \omega\right\rangle d y
$$

\footnotetext{
${ }^{1}$ Scuola Normale Superiore, P.zza dei Cavalieri 7, 56100 Pisa (Italy) delellis@cibs.sns.it
} 
for every smooth $m-n$ differential form $\omega$ (with $\omega_{n}$ we denote the Lebesgue measure of the unit ball in $\left.\mathbf{R}^{n}\right)$. If $u \in W^{1, n-1} \cap L^{\infty}$ and $F \in C^{1}\left(\mathbf{R}^{n}, \mathbf{R}^{n}\right)$ then

$$
\langle[J(F(u))], \omega\rangle=\frac{1}{\omega_{n}} \int_{\mathbf{R}^{n}} \operatorname{det} \nabla F(y)\left\langle\left[J u^{y}\right], \omega\right\rangle d y .
$$

It is natural to think of (2) as an extension of the chain rule for Jacobians of smooth maps, hence we will refer to it as weak chain rule. Of course when $u$ is smooth we have the much stronger relation $[J(F(u))]=\operatorname{det} \nabla F(u)[J u]$. For general $u$ we can not even give a meaning to the right hand side of this equation, because $[J u$ is only a distribution. However when $[J u]$ is a measure we might hope that there exists a pointwise representative $\bar{u}$ of $u$ which is Borel measurable and satisfies $[J(F(u))]=\operatorname{det} \nabla F(\bar{u})[J u]$ (where the right hand side is now well defined because $\operatorname{det} \nabla F(\bar{u})$ is a Borel function). In general this identity does not hold; if it holds we say that $u$ satisfies a strong chain rule.

If for every open set $A \subset \Omega$ we define

$$
\|J u\|(A):=\sup \left\{\langle[J u], \omega\rangle \mid \omega \text { is a } C_{c}^{\infty}(A) \text { form and }\|\omega\|_{\infty} \leq 1\right\},
$$

then a consequence of the previous Theorem is

$$
\|J u\|(A) \leq \frac{1}{\omega_{n}} \int_{\mathbf{R}^{n}}\left\|J u^{y}\right\|(A) d y .
$$

A natural question is to ask under what conditions the equality holds. When $n=1$ the problem reduces to the coarea formula on the level sets of a scalar function (see for example Theorem 3.40 of [1]). When $n>1$ Jerrard and Soner proved that the equality holds for $u \in W^{1, p}$ with $p>n$. In this case $[J u]$ is equal to the natural action as current of $d u_{1} \wedge \ldots \wedge d u_{n}$ and a comparison with the classical coarea formula for approximately differentiable functions (see for example Theorem 3.2.3 of [6]) implies that $\left[J u^{y}\right]$ is the integer rectifiable current supported by $u^{-1}(y)$ with the usual orientation. Motivated by these facts we will say that equation (1) represents a weak coarea formula and that $u$ satisfies the strong coarea formula if the equality holds in (3).

In general, for the validity of (3), it is not sufficient to assume merely that $\|J u\|$ is a Radon measure (see [9] for some counterexamples based on earlier ones of Giaquinta, Modica and Soucek [7]). However it is conjectured in [9] that the strong coarea formula holds for $W^{1, n}$ functions and for continuous functions such that $\|J u\|$ is a Radon measure.

In this paper we prove that if $u$ satisfies the strong chain rule then the strong coarea formula holds (see Theorem 3.1). This theorem provides a different proof of the strong coarea formula when $u \in W^{1, p}, p>n$, 
which can be extended to $u \in W^{1, n}$; more generally it implies that the strong coarea formula holds whenever $u$ is a Cartesian map in the sense of Giaquinta, Modica and Soucek (see Remarks 4.3 and 5.2).

In section 4 we prove (using arguments involving classical degree of continuous maps and distributional Jacobians) that if $u$ is a continuous BnV function in $W^{1, p}$ with $p>n-1$ and $\left\|J u^{y}\right\|(\Omega)$ is an integrable function of $y$ then $u$ satisfies the strong chain rule (see Theorem 4.1). This provides a partial answer to the conjecture of Jerrard and Soner. However we notice that in this case the strong coarea formula can be shown with a direct proof without passing through the strong chain rule. Unfortunately we are not able to prove that if $\left\|J u^{y}\right\|(\Omega) \notin L_{l o c}^{1}$ and $u$ is continuous then $\|J u\|(\Omega)=\infty$, which would prove the full conjecture in the case $p>n-1$.

Acknowledgments I wish to thank Professor Robert Jerrard for our discussions and his useful suggestions. This work was begun while the author enjoyed the hospitality of the Max Planck Institute for Mathematics in Leipzig.

\section{Preliminaries}

We begin this section by stating some basic definitions and properties of distributional Jacobians. We refer to [9] for a more general treatment and to [5] for definitions and proofs in the framework of Geometric measure theory. We notice that in [5] we used the "metric theory" of currents introduced by Ambrosio and Kirchheim in [2] because we think that it simplifies notations and proofs. However to make this paper more readable and self-contained we state definitions and theorems of this section in the language of "classical" Geometric measure theory.

We will denote by $\Lambda^{n}\left(\mathbf{R}^{m}\right)$ the vector space of $n$-covectors and we will endow it with the usual norm $|\cdot|$ as Hilbert space (see for example section 1 of [6]). If $\Omega \subset \mathbf{R}^{m}$ is an open set we will call $n$-form on $\Omega$ a function $\omega: \Omega \rightarrow \Lambda^{n}\left(\mathbf{R}^{m}\right)$. We will endow $C_{c}^{\infty}\left(\Omega, \Lambda^{n}\left(\mathbf{R}^{m}\right)\right)$ with the usual $C^{k}$ seminorms and we will call $n$-dimensional current on $\Omega$ a continuous linear functional on it. For the sake of simplicity when the regularity of a form $\omega$ is not specified we suppose that it is $C^{\infty}$. The exterior derivative on forms and the boundary operator on currents are defined as usual.

From now on $\Omega$ will denote an open set and $B_{r}(x)$ the open ball of radius $r$ centered on $x$. Moreover if $\mu$ is a Radon measure on $\Omega$ and $A \subset \Omega$ is a Borel set then $\mu \mathrm{L} A$ will indicate the measure $\nu$ given by $\nu(E)=\mu(A \cap E)$. 
Definition 2.1. If $T$ is a $k$-dimensional current on $\Omega$ and $A \subset \Omega$ is an open set then we define

$$
\|T\|_{h}(A):=\sup \left\{T(\omega) \mid \operatorname{supp}(\omega) \subset \subset A,\|\omega\|_{\infty} \leq 1\right\}
$$

If $\|T\|_{h}(\Omega)<\infty$ then $\|T\|_{h}$ can be extended to a Radon measure.

To simplify notations we will use $\|T\|$ in place of $\|T\|_{h}$ but we warn the reader that this notation is different from the one used in the literature. Indeed in the literature $\|T\|$ usually denotes the mass of $T$, which can be defined on open sets $A$ as

$$
\|T\|_{M}(A):=\sup \left\{T(\omega) \mid \operatorname{supp}(\omega) \subset \subset A,\|\omega\|_{\infty} \leq 1 \text { and } \omega \text { is simple }\right\} .
$$

The mass can be extended to a Radon measure when $\|T\|_{M}(\Omega)<\infty$. Moreover $\|T\|_{M} \leq\|T\|$ and there is a constant $k_{1}$ (which depends on the dimensions of $\Omega$ and of $T$ ) such that $\|T\| \leq k_{1}\|T\|_{M}$. Of course if $T$ is a 0 -dimensional current the two measures coincide.

Definition 2.2. Let us fix a simple $k$-form $\nu=u_{0} d u_{1} \wedge \ldots \wedge d u_{k}$ in $\Omega \subset \mathbf{R}^{m}$ with $u \in L^{\infty}, \nabla u_{i} \in L^{k}$ for every $i$ and $m \geq k$. We define the $m-k$ dimensional current $H_{\nu}$ via the action

$H_{\nu}\left(f d g_{1} \wedge \ldots \wedge d g_{m-k}\right)=\int_{\mathbf{R}^{m}} u_{0} f \operatorname{det}\left(\nabla g_{1}, \ldots, \nabla g_{m-k}, \nabla u_{1}, \ldots, \nabla u_{k}\right)$ extended to general differential forms by linearity.

Definition 2.3. Let $u \in W^{1, n-1}\left(\Omega, \mathbf{R}^{n}\right) \cap L^{\infty}$ with $\Omega \subset \mathbf{R}^{m}$. Then we define $j^{*}(u)$ as the $(n-1)$-form

$$
\sum_{i=1}^{n}(-1)^{i-1} u_{i} d u_{1} \wedge \ldots \wedge \widehat{d u}_{i} \wedge \ldots \wedge d u_{n}
$$

and $j(u)$ as the current on $\Omega$ given by $(-1)^{n} H_{j^{*}(u)}$. Moreover we call $[J u]:=\partial j(u) / n$ weak Jacobian of $u$ and we say that $u \in \mathrm{BnV}$ (or $u$ has bounded higher variation) if $\|J u\|(\Omega)$ is finite. In this paper to simplify the notation we will write Ju instead of [Ju].

Remark 2.4. We remark that $J u$ can be thought as the natural action as current of the distributional exterior derivative of $j^{*}(u) / n$. Indeed let us fix a system of coordinates in $\mathbf{R}^{m}$ and suppose that $j^{*}(u)=$ $\sum_{I \in J_{n-1}} f_{I} d x_{I}$ where:

(i) $J_{k}$ is the collection of all subsets of $\{1, \ldots, m\}$ with cardinality $k$;

(ii) $d x_{I}=d x_{i_{1}} \wedge \ldots \wedge d x_{i_{k}}$ if $I=\left\{i_{1}, \ldots, i_{k}\right\}$. 
Let us denote by $\partial_{j} f_{I}$ the partial derivative of $f_{I}$ with respect to $x_{j}$ in the sense of distributions and, given a distribution $T$, let us agree that the formal writing $S=T d x_{1} \wedge \ldots \wedge d x_{k}$ acts as a current via

$$
\left\langle S, f d g_{1} \wedge \ldots \wedge d g_{m-k}\right\rangle:=T\left(f \operatorname{det}\left(\nabla x_{1}, \ldots, \nabla x_{k}, \nabla g_{1}, \ldots, \nabla g_{m-k}\right)\right) .
$$

Hence it is not difficult to see that

$$
J u=\frac{1}{n} \sum_{I \in J_{n-1}} \sum_{j=1}^{m} \partial_{j} f_{I} d x_{I} \wedge d x_{j}:=\sum_{I \in J_{n}} T_{I} d x_{I} .
$$

Moreover $u$ is in $\mathrm{BnV}$ if and only if every $T_{I}$ is a measure with bounded variation. In this case $J u$ can be thought as a measure which takes values in the Hilbert space $\Lambda^{n}\left(\mathbf{R}^{m}\right)$ : in this setting $\|J u\|$ is exactly the total variation measure of $J u$.

When $u \in \mathrm{BnV}$ we can use the last remark to give a meaning to $\langle J u, \omega\rangle$ for every $\omega$ which is Borel measurable.

Theorem 2.5. Let us suppose that $u \in W^{1, n-1} \cap L^{\infty}\left(\Omega, \mathbf{R}^{n}\right)$, where $\Omega \subset \mathbf{R}^{n} \times \mathbf{R}^{m-n}$. If we put $v_{z}(w)=u(w, z)$ and $\Omega_{z}=\Omega \cap\left(\mathbf{R}^{n} \times\{z\}\right)$, then

$$
\left\langle J u, g d z_{1} \wedge \ldots \wedge d z_{m-n}\right\rangle=\int_{\mathbf{R}^{n}}\left\langle J v_{z}, g(\cdot, z)\right\rangle d z .
$$

Moreover if $u \in \mathrm{BnV}$ then $v_{z} \in \mathrm{BnV}\left(\Omega_{z}, \mathbf{R}^{n}\right)$ for a.e. $z$ and

$$
\|J u\|(A) \geq \int_{\mathbf{R}^{n}}\left\|J v_{z}\right\|\left(A \cap \Omega_{z}\right) d z
$$

for every measurable set $A \subset \Omega$.

We refer to [9] or to [5] for the proof. The next lemmas will focus on the relations between classical degree theory and weak Jacobians (see section 8 of [11]). If $\Omega \subset \mathbf{R}^{n}$ and $u \in C\left(\bar{\Omega}, \mathbf{R}^{n}\right)$ then we will denote by $\operatorname{deg}(u, \Omega, y)$ the degree of $u$ at $y$. If $M$ and $N$ are smooth compact manifolds of the same dimension and $u \in C(M, N)$ then we denote by $\operatorname{deg}(u, M, N)$ the degree of the map $u$.

Lemma 2.6. Let $u \in C^{\infty}\left(\Omega, \mathbf{R}^{n}\right)$, where $\Omega \subset \mathbf{R}^{n}$ has smooth boundary. Then for every vector field $\varphi \in C_{c}^{\infty}\left(\mathbf{R}^{n}, \mathbf{R}^{n}\right)$ we have

$$
\int_{\mathbf{R}^{n}} \nabla \cdot \varphi(y) \operatorname{deg}(u, \Omega, y) d y=\frac{1}{n} \int_{\partial \Omega} \varphi(u) \cdot M_{j}^{i}(\nabla u) \cdot \nu d \mathcal{H}^{n-1},
$$

where $\nu$ denotes the unit normal to $\partial \Omega$.

After a change of variables the proof of this Lemma follows from an integration by parts. 
Remark 2.7. When $\Omega \subset \mathbf{R}^{n}, j(u)$ can be seen as the vector field given by $v:=u \cdot M_{j}^{i}(\nabla u)$ (where $M_{j}^{i}$ is the cofactor matrix of $\nabla u$ ). Indeed let us take a form $\omega=g d x_{1} \wedge \ldots \wedge d x_{n-1}$ : then we have that

$$
\langle j(u), \omega\rangle=\int_{\mathbf{R}^{n}} g(x) v_{n}(x) d x .
$$

Hence the 0-current $J u$ is the divergence of $v / n$ in the sense of distributions.

Given a set $K \subset \mathbf{R}^{n}$ and a real number $\varepsilon>0$ we denote by $K_{\varepsilon}$ the set $\left\{x \in \mathbf{R}^{n} \mid \operatorname{dist}(x, K)<\varepsilon\right\}$.

Lemma 2.8. Let $u \in \operatorname{BnV}\left(\Omega, \mathbf{R}^{n}\right.$ ) be a continuous map (with $\Omega \subset \subset$ $\left.\mathbf{R}^{n}\right)$. For every $\varepsilon>0$ there exists an open set $M$ with smooth boundary such that

(a) $M \subset \Omega$ and $\Omega \subset M_{\varepsilon}$;

(b) $u \in W^{1, n-1}(\partial M)$;

(c) it holds

$$
\frac{1}{n} \int_{\partial M} u \cdot M_{j}^{i}(\nabla u) \cdot \nu d \mathcal{H}^{n-1}=J u(M) .
$$

Proof. First of all we choose an open set $M^{\prime} \subset \Omega$ with smooth boundary such that $\Omega \subset M_{\varepsilon / 2}^{\prime}$. A well known theorem on tubular neighborhoods implies that there exists a $\delta<\varepsilon / 2$ such that for every $0<t<\delta$ the sets

$$
S_{-t}=\left\{x \mid \operatorname{dist}\left(x, \partial M^{\prime}\right)=t\right\} \cap M^{\prime} \quad S_{t}=\left\{x \mid \operatorname{dist}\left(x, \partial M^{\prime}\right)=t\right\} \backslash M^{\prime}
$$

are smooth manifolds. Moreover we can define a signed distance function $d$ such that the sets $S_{-t}, S_{t}$ are the level sets of $d$. Hence for every $|s|<\delta, d^{-1}(s)$ is the boundary of an open set $M_{s}$ which satisfies (a). Applying the coarea formula to $d$ we conclude that for a.e. $s$ condition (b) holds for $M_{s}$.

Now let us put $v:=u \cdot M_{j}^{i}(\nabla u)$; we have $v \in L^{1}\left(\Omega, \mathbf{R}^{n}\right)$ and the measure $J u$ is the divergence of $v$ (see the previous Remark). Using standard approximation arguments we can find a sequence of $C^{\infty}$ vector fields $v_{n}$ such that, for a.e. $s, v_{n} \rightarrow v$ strongly in $L^{1}\left(\partial M_{s}\right)$ and

$$
\frac{1}{n} \int_{M_{s}} \nabla \cdot v_{n} \rightarrow J u\left(M_{s}\right) \text {. }
$$

Hence it follows that condition (c) is satisfied by $M_{s}$ for a.e. $s$.

Lemma 2.9. If $M \subset \subset \mathbf{R}^{n}$ is an open set with smooth boundary and $u \in W^{1, n-1}\left(\partial M, S^{n-1}\right)$ is continuous then

$$
\omega_{n} \operatorname{deg}\left(u, \partial M, S^{n-1}\right)=\frac{1}{n} \int_{\partial M} u \cdot M_{j}^{i}(\nabla u) \cdot \nu d \mathcal{H}^{n-1} .
$$


Proof. A straightforward calculation shows that $\frac{1}{n} u \cdot M_{j}^{i}(\nabla u) \cdot \nu$ is the Jacobian determinant of $u$ as map from $\partial M$ to $S^{n-1}$. Then the statement of the Lemma follows from the integral formula for the degree as can be found for example in Brezis and Nirenberg [3].

Lemma 2.10. Let us suppose that $u \in C\left(\bar{\Omega}, \mathbf{R}^{n}\right) \cap W^{1, n-1}$, where $\Omega \subset$ $\mathbf{R}^{n}$. Then for a.e. $y \in \mathbf{R}^{n}$ we have

$$
\omega_{n}|\operatorname{deg}(u, \partial \Omega, y)| \leq\left\|J u^{y}\right\|(\Omega) .
$$

Proof. As it is stated in Theorem 1.1, $u^{y} \in W^{1, n-1}\left(\Omega, S^{n-1}\right)$ for a.e. $y$ (see Lemma 6 in sec. 4 of [9] for a proof of this fact). If $y \in u(\partial \Omega)$ then $|\operatorname{deg}(u, \partial \Omega, y)|=0$ and the statement is obviously true; in an analogous way we reason if $\left\|J u^{y}\right\|(\Omega)=\infty$. Hence let us fix a $y \notin u(\partial \Omega)$ such that $u^{y} \in W^{1, n-1}\left(\Omega, S^{n-1}\right)$ and $\left\|J u^{y}\right\|(\Omega)<\infty$. If we take an open set $M$ with smooth boundary such that $u^{y} \in W^{1, n-1} \cap C(\partial M)$ then

$$
\omega_{n} \operatorname{deg}\left(u^{y}, \partial M, S^{n-1}\right)=\frac{1}{n} \int_{\partial M} u^{y} \cdot M_{j}^{i}\left(\nabla u^{y}\right) \cdot \nu d \mathcal{H}^{n-1} .
$$

Using Lemma 2.8 we conclude that for $\varepsilon$ sufficiently small there is an open set $M$ such that $M \subset \Omega \subset M_{\varepsilon}, u^{y} \in W^{1, p} \cap C(\partial M)$ and

$$
\omega_{n} \operatorname{deg}\left(u^{y}, \partial M, S^{n-1}\right)=J u^{y}(M) .
$$

We notice that for $\varepsilon$ small enough we have

$$
\operatorname{deg}\left(u^{y}, \partial M, S^{n-1}\right)=\operatorname{deg}(u, \partial M, y)=\operatorname{deg}(u, \partial \Omega, y)
$$

and this completes the proof.

We end this section with a corollary of the area formula (see [10] or Corollary 3.2.20 in [6] for the proof).

Lemma 2.11. Let $\Gamma$ be an oriented $C^{\infty}(n-1)$-dimensional manifold and suppose that $u \in W^{1, p}\left(\Gamma, \mathbf{R}^{n}\right) \cap C$ with $p>n-1$. Then for any $\mathcal{H}^{n-1}$ measurable set $A \subset \Gamma$ we have

$$
\mathcal{H}^{n-1}(u(A)) \leq(n-1)^{(1-n) / 2} \int_{A}|D u|^{n-1} d \mathcal{H}^{n-1}
$$

\section{Strong COAREA FORMUla}

In this section we prove that the strong chain rule implies the strong coarea formula. In particular we have the following

Theorem 3.1. Let $u \in \operatorname{BnV}\left(\Omega, \mathbf{R}^{n}\right)$, where $\Omega \subset \mathbf{R}^{m}$, and suppose that there is a pointwise representative $\bar{u}$ of $u$ such that $J(F(u))=$ $\operatorname{det} \nabla F(\bar{u})$ Ju for every $F \in C^{1}\left(\mathbf{R}^{n}, \mathbf{R}^{n}\right)$. Then

$$
\omega_{n} \int_{\mathbf{R}^{n}}\left\|J u^{y}\right\|(\Omega)=\|J u\|(\Omega) .
$$


In the following we deal with family of currents parameterized by variables in $\mathbf{R}^{n}$ : for the sake of clarity if $T_{y}$ is a family of currents on $\mathbf{R}^{m}$ parameterized by $y$ and $\omega$ is a form sometimes we will indicate with $\left\langle T_{y}(x), \omega(x)\right\rangle$ the real numbers $T_{y}(\omega)$.

Proof. To simplify notations we will identify $u$ and $\bar{u}$. Moreover we notice that it is sufficient to prove

$$
\|J u\|(\Omega) \geq \frac{1}{\omega_{n}} \int_{\mathbf{R}^{n}}\left\|J u^{y}\right\|(\Omega) d y .
$$

Let us define the current $T$ on $\Omega_{x} \times \mathbf{R}_{y}^{n}$ in the following way:

(a) if $\omega=\sum f_{I}(x, y) d x_{I}$ then

$$
T(\omega)=\frac{1}{\omega_{n}} \int_{\mathbf{R}^{n}} \sum\left\langle J u^{y}, f_{I}(x, y) d x_{I}\right\rangle d y ;
$$

(b) if $\omega=f(x, y) \nu \wedge d y_{j}$ then $\langle T, \omega\rangle=0$;

(c) $T$ is extended by linearity to the whole space of smooth forms.

(In more "technical" words we have

$$
T(\omega)=\frac{1}{\omega_{n}} \int_{\mathbf{R}^{n}}\left\langle\overline{J u}_{y}, \omega\right\rangle d y
$$

where $\overline{J u}_{y}$ is the push-forward of $J u^{y}$ via the injection $i_{y}: \Omega \rightarrow \Omega \times \mathbf{R}^{n}$ given by $i_{y}(x)=(x, y)$.) We will prove that

$$
\|J u\|(\Omega) \geq\|T\|\left(\Omega \times \mathbf{R}^{n}\right) \geq \frac{1}{\omega_{n}} \int_{\mathbf{R}^{n}}\left\|J u^{y}\right\|(\Omega) .
$$

Step 1 We prove the second inequality of (7). First of all we recall that

$$
\int_{\mathbf{R}^{n}}\left\|j\left(u^{y}\right)\right\|_{L^{1}} d y=\omega_{n}\|j(u)\|_{L^{1}}<\infty .
$$

Let $W^{\prime}$ be the set of points $y_{0}$ such that

$$
\lim _{\varepsilon \rightarrow 0} \frac{1}{\omega_{n} \varepsilon^{n}} \int_{B_{\epsilon}\left(y_{0}\right)}\left\|j\left(u^{y_{0}}\right)-j\left(u^{y}\right)\right\|_{L^{1}} d y=0 .
$$

Then equation (8) gives that $\mathcal{L}^{n}\left(\mathbf{R}^{n} \backslash W^{\prime}\right)=0$. We claim that, fixed an $\eta>0$, for every $z \in W^{\prime}$ there is an $\varepsilon(z)$ such that

(A) For every $\delta<\varepsilon(z)$ there is a smooth form $\nu$ such that $\|\nu\|_{\infty} \leq 1$, $\operatorname{supp}(\nu) \subset \Omega \times B_{\delta}(z)$ and $T(\nu) \geq(1-\eta)^{3} \delta^{n}\left\|J u^{z}\right\|(\Omega)$.

Using (A) and standard covering arguments it is easy to find a sequence of smooth $n$-forms $\nu_{k}(x, y)$ on $\Omega \times \mathbf{R}^{n}$ such that $\left\|\nu_{k}\right\|_{\infty} \leq 1$ and

$$
\|T\|\left(\Omega \times \mathbf{R}^{n}\right) \geq \limsup _{k \rightarrow \infty} T\left(\omega_{k}\right) \geq(1-\eta)^{3} \frac{1}{\omega_{n}} \int_{\mathbf{R}^{n}}\left\|J u^{y}\right\|(\Omega) d y .
$$


If we let $\eta$ go to 0 we have the desired inequality.

To prove (A) we reason as follows. First of all we find a smooth $\omega \in C_{c}^{\infty}\left(\Omega, \Lambda^{m-n}\left(\mathbf{R}^{m}\right)\right)$ such that $\|\omega\|_{\infty} \leq 1$ and $\left\langle J u^{z}, \omega\right\rangle \geq(1-$ $\eta)\left\|J u^{z}\right\|(\Omega)$. Then we notice that there is an $\varepsilon$ such that for $\delta \leq \varepsilon$

$$
\begin{aligned}
\frac{1}{\omega_{n} \delta^{n}} \int_{B_{\delta}(z)}\left\langle J u^{y}(x), \omega(x)\right\rangle d y & =\frac{1}{\omega_{n} \delta^{n}} \int_{B_{\delta}(z)}\left\langle j\left(u^{y}\right)(x), d \omega(x)\right\rangle d y \\
& \geq(1-\eta)\left\langle j\left(u^{z}\right), d \omega\right\rangle=(1-\eta)\left\langle J u^{z}, \omega\right\rangle .
\end{aligned}
$$

Moreover with the same idea it is not difficult to find for every $\delta$ a function $\varphi \in C_{c}^{\infty}\left(B_{\delta}(z)\right)$ such that

$$
\int_{\mathbf{R}^{n}}\left\langle J u^{y}(x), \varphi(y) \omega(x)\right\rangle d y \geq(1-\eta) \int_{B_{\delta}(z)}\left\langle J u^{y}, \omega\right\rangle d y .
$$

Then the form $\nu(x, y)=\varphi(y) \omega(x)$ satisfies condition (A) for $\delta$.

Step 2 We will prove that $\|T\|\left(\Omega \times \mathbf{R}^{n}\right) \leq\|J u\|(\Omega)$. First we notice that the strong chain-rule and Theorem 4.1 imply

$$
\begin{aligned}
\langle\operatorname{det} \nabla F(y) \omega(x) & T(x, y)\rangle=\frac{1}{\omega_{n}} \int_{\mathbf{R}^{n}} \operatorname{det} \nabla F(y)\left\langle J u^{y}(x), \omega(x)\right\rangle d y \\
& =\langle[\operatorname{det} \nabla F(u(x))] \omega(x), J u(x)\rangle
\end{aligned}
$$

for every $\omega$ with $\operatorname{supp}(\omega) \subset \subset \Omega$ and for every $F \in C^{1}\left(\mathbf{R}^{n}, \mathbf{R}^{n}\right)$. Now let us take a real function $\psi \in C^{1}\left(\mathbf{R}^{n}\right)$ : it is easy to see that we can find two functions $\psi_{1}, \psi_{2}$ such that $\psi_{1}+\psi_{2}=\psi, \psi_{i} \in C^{1}$ and $\psi_{1} \geq k$, $\psi_{2} \leq-k$ for some $k>0$. From a result of Dacorogna and Moser (see [4]) it follows that for every ball $B \subset \mathbf{R}^{n}$ we can find functions $F_{B}^{i} \in C^{1}\left(\mathbf{R}^{n}, \mathbf{R}^{n}\right)$ such that

$$
\operatorname{det} \nabla F_{B}^{i}(y)=\psi_{i}(y) \quad \text { for every } y \in B \text {. }
$$

If we fix a form $\omega$ with compact support we can find a ball $B$ such that $u(\operatorname{supp}(\omega)) \subset B$, hence equation (9) applied to $F_{B}^{i}$ and the multilinearity of $T$ give

$$
\langle\psi(y) \omega(x), T(x, y)\rangle=\langle\psi(u(x)) \omega(x), J u(x)\rangle .
$$

Now we would like to use density arguments to find:

$$
\langle\psi(x, y) \omega(x), T(x, y)\rangle=\langle\psi(x, u(x)) \omega(x), J u(x)\rangle .
$$

for every $\psi \in C_{c}^{1}\left(\Omega \times \mathbf{R}^{n}\right)$. We notice that (8) and the relation $\partial j\left(u^{y}\right)=$ $J u^{y}$ imply

$$
\lim _{k \rightarrow \infty} T\left(\psi_{k}(x, y) \omega(x)\right)=T(\psi(x, y) \omega(x))
$$


if $\psi_{k} \rightarrow \psi$ in $C^{1}$. Moreover we remark that the vector space generated by

$$
\left\{\phi \in C_{c}^{1}\left(\Omega \times \mathbf{R}^{n}\right) \mid \phi \text { is of the form } g(x) f(y)\right\}
$$

is dense in $C_{c}^{1}\left(\Omega \times \mathbf{R}^{n}\right)$ in the strong topology. These facts imply that (10) holds.

By the definition of $T$ we have that if $\nu$ is a smooth form on $\Omega \times \mathbf{R}^{n}$ with compact support then there are a scalar function $\psi \in C^{\infty}\left(\Omega \times \mathbf{R}^{n}\right)$ and an $(m-n)$-form $\omega$ on $\Omega$ such that

(a) $T(\nu)=T(\psi(x, y) \omega(x))$;

(b) $\|\psi \omega\|_{\infty} \leq\|\nu\|_{\infty}$.

We conclude that $(10)$ gives $\|T\|\left(\Omega \times \mathbf{R}^{n}\right) \leq\|J u\|(\Omega)$.

\section{Strong Chain RUle}

In this section we will prove the following

Theorem 4.1. Let $u \in W^{1, p}\left(\Omega, \mathbf{R}^{n}\right)$ be continuous, with $\Omega \subset \mathbf{R}^{m}$ and $p>n-1$. Moreover suppose that

$$
\int_{\mathbf{R}^{n}}\left\|J u^{y}\right\|(\Omega) d y<\infty .
$$

If $F \in C^{1}\left(\mathbf{R}^{n}, \mathbf{R}^{n}\right)$ then $F(u) \in \operatorname{BnV}$ and $J(F(u))=\operatorname{det} \nabla F(u) J u$.

First of all we will prove the Theorem when $m=n$ and then we will recover the general case with a slicing argument. The key ingredient for the case $m=n$ is the lemma stated below, where we use some ideas of Müller and Spector (see sec. 8 of [11]). We remark that the hypothesis $p>n-1$ is needed in the proof of the first step of Lemma 4.2. Indeed in this proof we use Lemma 2.11 which in general does not hold when $p=n-1$.

The continuity of $u$ is used heavily in the second step of Lemma 4.2 and the technical hypothesis (11) is employed to derive condition (C) below. Condition (C) is crucial for passing to the limit in formula (15) because it allows to use the Dominated Convergence Theorem. Indeed it could be true that the relation

$$
\int_{\mathbf{R}^{n}} \operatorname{deg}(u, A, y) d y=J u(A)
$$

holds true for every continuous BnV function $u$ and for every open set $A$ such that $\mathcal{L}^{n}(u(A))=0$. This would imply that the strong chain rule, and hence the strong coarea formula, are true for every continuous $\mathrm{BnV}$ function in $W^{1, p}, p>n-1$. However at the present stage we do not know how to derive equality (12) without using condition (C). 
Lemma 4.2. Let $u \in \operatorname{BnV}\left(\Omega, \mathbf{R}^{n}\right) \cap W^{1, p}$, where $\Omega \subset \mathbf{R}^{n}$ and $p>$ $n-1$. Suppose that $u$ is continuous and that there exists a function $N \in L_{l o c}^{1}\left(\mathbf{R}^{n}\right)$ such that

(C) for all open $\Omega^{\prime} \subset \subset \Omega,\left|\operatorname{deg}\left(u, y, \Omega^{\prime}\right)\right| \leq N(y)$ for a.e. $y$.

Then for every open set $A \subset \subset \Omega$ such that $\mathcal{L}^{n}(u(\partial A))=0$ we have

$$
\int_{\mathbf{R}^{n}} \operatorname{deg}(u, y, A) d y=J u(A), \quad \int_{\mathbf{R}^{n}}|\operatorname{deg}(u, y, A)| d y \leq\|J u\|(A) .
$$

Proof. We split the proof into two steps.

First step We prove the first equation of (13) when $A$ is an open set with smooth boundary such that $u \in W^{1, p}(\partial A)$ and

$$
\frac{1}{n} \int_{\partial A} u \cdot M_{j}^{i}(\nabla u) \cdot \nu d \mathcal{H}^{n-1}=J u(A) .
$$

Let us approximate $u$ strongly in $L^{\infty}$ and in $W^{1, p}(A) \cap W^{1, p}(\partial A)$ with $C^{\infty}$ functions $u_{k}$. The formula of integration by parts of Lemma 2.6 gives

$$
\int_{\mathbf{R}^{n}} \nabla \cdot \varphi(y) \operatorname{deg}\left(u_{k}, A, y\right) d y=\frac{1}{n} \int_{\partial A} \varphi(u) \cdot M_{j}^{i}\left(\nabla u_{k}\right) \cdot \nu d \mathcal{H}^{n-1}
$$

for every $C_{c}^{\infty}$ vector field. This equation implies that $f_{k}:=\operatorname{deg}\left(u_{k}, A, y\right)$ are functions of bounded variation and

$$
\left\|D f_{k}\right\|\left(\mathbf{R}^{n}\right) \leq \frac{1}{n} \int_{\partial A}\left\|M_{j}^{i}\left(\nabla u_{k}\right)\right\| d \mathcal{H}^{n-1} \leq \frac{1}{n}\left\|\nabla u_{k}\right\|_{L^{n-1}(\partial A)}^{n-1} .
$$

Moreover the supports of $f_{k}$ are all contained in a ball big enough. From the Poincaré inequality for BV functions it follows that their BV norms are equibounded, hence the sequence $f_{k}$ is weakly compact in $L^{1}$. Moreover $\operatorname{deg}\left(u_{k}, A, y\right)$ converges to $\operatorname{deg}(u, A, y)$ for every $y \notin u(\partial A)$ and Lemma 2.11 implies that $u(\partial A)$ is negligible, hence we have $f_{k}=$ $\operatorname{deg}\left(u_{k}, A, y\right) \rightarrow \operatorname{deg}(u, A, y)$ strongly in $L^{1}$. Passing to the limit we conclude that

$$
\int_{\mathbf{R}^{n}} \operatorname{deg}(u, y, A)=\frac{1}{n} \int_{\partial A} u \cdot M_{j}^{i}(\nabla u) \cdot \nu d \mathcal{H}^{n-1}
$$

and from (14) we obtain the first equation of (13).

Second Step Let us fix an open set $A$ with $\mathcal{L}^{n}(u(\partial A))=0$ : using Lemma 2.8 we choose open sets $A_{k} \uparrow A$ such that every $A_{k}$ has smooth boundary and satisfies (14). By classical arguments we have $\operatorname{deg}\left(u, A_{k}, y\right) \rightarrow \operatorname{deg}(u, A, y)$ for a.e. $y$ and the Dominated convergence Theorem allows us to conclude

$$
\int_{\mathbf{R}^{n}} \operatorname{deg}(u, A, y) d y=\lim _{k \rightarrow \infty} \int_{\mathbf{R}^{n}} \operatorname{deg}\left(u, A_{k}, y\right) d y
$$




$$
=\lim _{k \rightarrow \infty} J u\left(A_{k}\right)=J u(A) .
$$

Now let us define for every $k \in \mathbb{Z}$

$$
L_{k}:=\{y \mid \operatorname{deg}(u, y, A)=k\} \quad B_{k}=u^{-1}\left(L_{k}\right) \cap A .
$$

Then we have that every $L_{k}$ is an open set and its boundary is contained in $u(\partial A)$. It follows that every $B_{k}$ is an open set and $u\left(\partial B_{k}\right)$ has measure zero. Moreover $|\operatorname{deg}(u, y, A)|=\sum_{k}\left|\operatorname{deg}\left(u, y, B_{k}\right)\right|$ and we conclude

$$
\int_{\mathbf{R}^{n}}|\operatorname{deg}(u, y, A)| d y=\sum_{k}\left|J u\left(B_{k}\right)\right| \leq\|J u\|(A) .
$$

\section{Proof of Theorem 4.1 First step: $\Omega \subset \mathbf{R}^{n}$.}

Equation (11), the weak coarea formula and the weak chain rule imply that $u$ and $F(u)$ are $B n V$ function, hence the writing $\operatorname{det} \nabla F(u) J u$ is formally correct in the distributional sense because it is the product of a measure by a continuous function. Moreover condition (11) implies condition (C) of the previous Lemma (see Lemma 2.10). Then, thanks to standard measure-theoretic arguments, we only have to check

$$
J(F(u))(B)=\int_{B} \operatorname{det} \nabla F(u(x)) d J u(x) .
$$

for every ball $B \subset \subset \Omega$.

We notice that the previous lemma implies

$$
J(F(u))(B)=\int_{\mathbf{R}^{n}} \operatorname{deg}(F(u), y, B) d y=\int_{\mathbf{R}^{n}} \operatorname{det} \nabla F(y) \operatorname{deg}(u, y, B) d y .
$$

By standard covering arguments and the continuity of $u$ we can find a countable family of pairwise disjoint balls $B_{i}$ centered on $x_{i}$ such that

(a) $\|J u\|(B)=\sum_{i}\|J u\|\left(B_{i}\right)$;

(b) $\|J(F(u))\|(B)=\sum_{i}\|J(F(u))\|\left(B_{i}\right)$;

(c) $\left|\operatorname{det} \nabla F(y)-\operatorname{det} \nabla F\left(u\left(x_{i}\right)\right)\right| \leq \varepsilon$ for every $y \in u\left(B_{i}\right)$.

First of all we notice that for a.e. $y$

$$
\operatorname{deg}(u, y, B)=\sum_{i} \operatorname{deg}\left(u, y, B_{i}\right) .
$$

Indeed Lemma 2.11 implies $\mathcal{L}^{n}\left(u\left(\partial B_{i}\right)\right)=0$, hence we have

$$
\int_{\mathbf{R}^{n}}\left|\operatorname{deg}(u, y, B)-\sum_{i=1}^{N} \operatorname{deg}\left(u, y, B_{i}\right)\right| d y
$$




$$
\int_{\mathbf{R}^{n}}\left|\operatorname{deg}\left(u, y, B \backslash \bigcup_{i=1}^{N} \bar{B}_{i}\right)\right| \leq\|J u\|\left(B \backslash \bigcup_{i=1}^{N} \bar{B}_{i}\right)
$$

and if we let $N$ go to infinity condition (b) gives (16). Moreover conditions (b) and (c) imply that

$$
\begin{gathered}
\Delta_{i}:=\left|\operatorname{det} \nabla F\left(u\left(x_{i}\right)\right) J u\left(B_{i}\right)-\int_{\mathbf{R}^{n}} \operatorname{deg}\left(F(u), y, B_{i}\right) d y\right| \\
\leq \int\left|\operatorname{det} \nabla F(y)-\operatorname{det} \nabla F\left(u\left(x_{i}\right)\right)\right|\left|\operatorname{deg}\left(u, y, B_{i}\right)\right| d y \leq \varepsilon\|J u\|\left(B_{i}\right)
\end{gathered}
$$

and that

$$
\Lambda_{i}:=\left|\operatorname{det} \nabla F\left(u\left(x_{i}\right)\right) J u\left(B_{i}\right)-\int_{B_{i}} \operatorname{det} \nabla F(u(x)) d J u(x)\right| \leq \varepsilon\|J u\|\left(B_{i}\right) .
$$

Hence we have

$$
\begin{aligned}
& \left|J(F(u))(B)-\int_{B} \operatorname{det} \nabla F(u(x)) d J u(x)\right| \\
\leq & \sum_{i}\left|J(F(u))\left(B_{i}\right)-\int_{B_{i}} \operatorname{det} \nabla F(u(x)) d J u(x)\right| \\
\leq & \sum_{i}\left(\Delta_{i}+\Lambda_{i}\right) \leq 2 \varepsilon \sum_{i}\|J u\|\left(B_{i}\right)=2 \varepsilon\|J u\|(B) .
\end{aligned}
$$

If we let $\varepsilon$ go to zero we conclude $J(F(u))(B)=[\operatorname{det} \nabla F(u) J u](B)$.

Second Step Again as in the previous step we remark that both $F(u)$ and $u$ are functions of bounded higher variation. For sake of simplicity we choose an orthogonal system of coordinates and we denote by $x_{1}, \ldots, x_{m-n}$ the first $m-n$ and by $z_{1}, \ldots, z_{n}$ the remaining ones. Moreover let us fix a form $\omega$ of type $f d x_{1} \wedge \ldots \wedge d x_{m-n}$ and define $v_{x}(z):=u(x, z), \Omega_{x}:=\Omega \cap\left(\{x\} \times \mathbf{R}^{n}\right)$. Of course we have $v_{x}^{y}(z)=$ $u^{y}(x, z)$, hence applying Theorem 2.5 and Fubini Tonelli Theorem we have

$$
\begin{aligned}
\int_{\mathbf{R}^{m-n}} \int_{\mathbf{R}^{n}}\left\|J v_{x}^{y}\right\|\left(\Omega_{x}\right) d y d x & =\int_{\mathbf{R}^{n}} \int_{\mathbf{R}^{m-n}}\left\|J v_{x}^{y}\right\|\left(\Omega_{x}\right) d x d y \\
& \leq \int_{\mathbf{R}^{n}}\left\|J u^{y}\right\|(\Omega) d y<\infty
\end{aligned}
$$

It follows that for a.e. $x$

$$
\int_{\mathbf{R}^{n}}\left\|J v_{x}^{y}\right\|\left(\Omega_{x}\right) d y<\infty .
$$


From the slicing arguments and the previous step we know that

$$
\begin{aligned}
\langle J(F(u)), \omega\rangle & =\int_{\mathbf{R}^{m-n}}\left\langle f(\cdot, x), J\left(F\left(v_{x}\right)\right\rangle d x\right. \\
& =\int_{\mathbf{R}^{m-n}}\left\langle\operatorname{det} \nabla F\left(v_{x}\right) f(\cdot, x), J v_{x}\right\rangle d x \\
& =\langle J u, \omega \operatorname{det} \nabla F(u)\rangle .
\end{aligned}
$$

We can reason in the same way for every differential form $\omega=f d \pi$, where $\pi$ is a projection on $m-n$ coordinates, and this completes the proof.

Remark 4.3. If $u \in W^{1, p}\left(\Omega, \mathbf{R}^{n}\right)$ with $p \geq n$ then the strong chain rule holds without any other assumption. For sake of simplicity let us suppose $\Omega \subset \mathbf{R}^{n}$. If we approximate $u$ strongly in $W^{1, p}$ with $C^{\infty}$ functions $u_{k}$ we have that $J u=\operatorname{det} \nabla u \mathcal{L}^{n}\left\llcorner\Omega\right.$ and $\operatorname{det} \nabla u_{k} \rightarrow \operatorname{det} \nabla u$ strongly in $L^{p / n}$. Moreover if $F \in C^{1}\left(\mathbf{R}^{n}, \mathbf{R}^{n}\right)$, then

$$
J\left(F\left(u_{k}\right)\right)=\operatorname{det} \nabla F\left(u_{k}\right) \operatorname{det} \nabla u_{k} \mathcal{L}^{n}\llcorner\Omega .
$$

But $J\left(F\left(u_{k}\right)\right) \rightarrow J(F(u))$ and passing to the limit we have $J(F(u))=$ $\operatorname{det} \nabla F(u) J u$. In the same way we can recover the general case $\Omega \subset \mathbf{R}^{m}$ with $m \geq n$.

\section{FURTHER REMARKS}

As corollary of Theorem 3.1 and of Theorem 4.1 we have

Theorem 5.1. Let $u \in W^{1, p}\left(\Omega, \mathbf{R}^{n}\right)$, where $\Omega \subset \mathbf{R}^{m}$ and $p>n-1$. If $u$ is continuous and $\left\|J u^{y}\right\|(\Omega) \in L^{1}\left(\mathbf{R}^{n}\right)$ then the strong coarea formula holds.

However a direct proof of Theorem 5.1 can be given as follows. First of all we can observe that

$$
\int_{u^{-1}(B)} \varphi d J u=\int_{B}\left\langle J u^{y}, \varphi\right\rangle d y
$$

for every $m-n$ form $\varphi \in C_{c}^{1}\left(u^{-1}(B)\right)$ and for every ball $B$. Hence using the condition $\left\|J u^{y}\right\|(\Omega) \in L^{1}$ and an approximation argument we can argue that the same formula holds for every form $\varphi \in C\left(u^{-1}(\bar{B})\right)$. At this point using an argument similar to that of step 1 of the proof of Theorem 3.1 we get the strong coarea formula.

Remark 5.2. Another interesting consequence of Theorem 3.1 is the following. Let us suppose that $u \in W^{1, p} \cap L^{\infty}$ is a Cartesian map, i.e. the graph of $u$ is a rectifiable current which has no boundary in the interior of $\Omega \times \mathbf{R}^{n}$ (see [8] for the theory of Cartesian maps and 
related questions). Then it is easy to prove that $\|J u\|$ is an absolutely continuous measure and that its density coincides with the pointwise Jacobian. Hence using the invariance of the definition of Cartesian map by composition of smooth maps we point out that

$$
J(F(u))=\operatorname{det} \nabla F(u) J u \quad \text { for every } F \in C^{1}\left(\mathbf{R}^{n}, \mathbf{R}^{n}\right) .
$$

Applying Theorem 3.1 we conclude that the strong coarea formula holds for $u$. Moreover for Sobolev functions we have a classical coarea formula involving the pointwise Jacobian (see [8] or Theorem 3.2.3 of [6]): a comparison gives that if $u$ is a Cartesian map then there exists a set $\Omega^{\prime} \subset \Omega$ of full measure such that $\left\|J u^{y}\right\|=\mathcal{H}^{m-n}\left\llcorner\left(u^{-1}(y) \cap \Omega^{\prime}\right)\right.$ for a.e. $y$.

Finally we notice that Remark 4.3 and Theorem 3.1 give a proof of the strong coarea formula in the case of $u \in W^{1, p}$ with $p>n$ which is different from the one given in [9] and can be extended to the case $p=n$.

\section{REFERENCES}

[1] Ambrosio L., Fusco N., Pallara D. Functions of bounded variation and free discontinuity problems. Oxford Mathematical Monographs. Clarendon Press, Oxford, 2000.

[2] Ambrosio L., KirchHeim B. Currents on metric spaces. Acta Mathematica, 185(1):1-80, 2000.

[3] Brezis H., Nirenberg L. Degree theory and BMO: Part 1, compact manifolds without boudaries. Selecta Math. (N.S.), 1(2):197-263, 1995.

[4] Dacorogna B., Moser J. On a partial differential equation involving the jacobian determinat. Ann. IHP Anal. Non Lin., 7(1):1-26, 1990.

[5] DE Lellis C. Fine properties of currents and applications to distributional Jacobians. To appear in Proc. Roy. Soc. Edinburgh Sect. A.

[6] Federer H. Geometric measure theory. Classics in Mathematics. Springer Verlag, Berlin, 1969.

[7] Giaquinta M., Modica G., SoučEk J. Cartesian currents, weak diffeomorphisms and existence theorems in nonlinear elasticity. Arch. Rat. Mech. Anal., 106(2):97-159, 1989.

[8] Giaquinta M., Modica G., SoučEK J. Cartesian currents in the calculus of variations, volume 1, 2. Springer Verlag, Berlin, 1998.

[9] Jerrard L., Soner M. Functions of higher bounded variation. Forthcoming.

[10] Marcus M., Mizel V. J. Transformations by functions in Sobolev spaces and lower semicontinuity for parametric variational integrals. Bull. Amer. Math. Soc., 79:790-795, 1973.

[11] Müller S., SPECTOR S. An existence theory for nonlinear elasticity that allows for cavitation. Arch. Rat. Mech. Anal., 131(1):1-66, 1995. 